\title{
Ansiedade, Avaliação Cognitiva e Esgotamento na Formação Desportiva: Estudo com Jovens Atletas
}

\author{
Anxiety, Cognitive Appraisal and Burnout in Sport: A Study with Young \\ Athletes
}

Carla Vilela ${ }^{1}$, A. Rui Gomes ${ }^{1 *}$

ARTIGO ORIGINAL | ORIGINALARTICLE

\begin{abstract}
Este estudo analisa a importância dos processos de avaliação cognitiva na experiência de ansiedade e de burnout bem como a importância da avaliação cognitiva e da ansiedade na predição do burnout. Participaram nesta investigação 711 atletas (89 do sexo feminino e 622 do sexo masculino), com idades compreendidas entre os 12 e os 19 anos $(M=14.77 ; D P=1.86)$. Os participantes responderam a um protocolo de avaliação composto por três instrumentos, além de um questionário demográfico: Escala de Ansiedade no Desporto-2; Escala de Avaliação Cognitiva e Questionário de Burnout para Atletas. Os resultados demonstraram que a avaliação cognitiva primária desempenha um papel fundamental na experiência de ansiedade e de burnout, dado que a perceção de ameaça correspondeu a maiores níveis de ansiedade e burnout e a perceção de desafio correspondeu a menores níveis nestas variáveis. Além disso, verificou-se que as dimensões da avaliação cognitiva primária, em conjunto com as dimensões da ansiedade, foram variáveis preditoras do burnout. Em suma, os resultados evidenciaram a importância da avaliação cognitiva no estudo dos estados emocionais dos atletas, sugerindo a continuação de investigações futuras neste domínio.
\end{abstract}

Palavras-chave: Ansiedade-Traço, Avaliação Cognitiva, Burnout, Desporto Juvenil.

\begin{abstract}
This study analyzed the importance of cognitive appraisal in the experience of anxiety and burnout as well as the importance of cognitive appraisal and anxiety in the prediction of burnout. The study included 711 athletes (89 females and 622 males), with ages between 12 and 19 years old $(M=14.77 ; D P=1.86)$. The participants answered an assessment protocol that included the Sport Anxiety Scale-2; the Cognitive Appraisal Scale and the Athlete Burnout Questionnaire, besides a demographic questionnaire. The results showed that primary cognitive appraisal influence the experience of anxiety and burnout, because athletes with higher levels of threat perception assumed more anxiety and burnout, and athletes with higher levels of challenge perception assumed lower levels of anxiety and burnout. The results also showed that the dimensions of primary cognitive appraisal and anxiety predicted the experience of athletes' burnout. In sum, this study highlights the importance of cognitive appraisal in the study of athletes' emotional states, suggesting the continuation of future research on this topic.

Keywords: Trait-Anxiety, Cognitive Appraisal, Burnout, Youth Sport.
\end{abstract}

Artigo recebido a 22.07.2014; Aceite a 21.07.2015

${ }^{1}$ Universidade do Minho, Portugal

* Autor correspondente: Universidade do Minho. Escola de Psicologia. Campus de Gualtar. 4710-057 Braga, Portugal. E-mail: rgomes@psi.uminho.pt 


\section{INTRODUÇÃO}

A formação desportiva está associada a inúmeros aspetos positivos do desenvolvimento dos atletas (Holt \& Neely, 2011). Jovens que praticam desporto apresentam níveis mais elevados de autoestima, regulação emocional, competências de resolução de problemas, realização de objetivos e competências sociais, quando comparados a jovens que não fazem desporto organizado (Holt \& Neely, 2011). Esta atividade permite que os jovens aprendam a competir e a cooperar uns com os outros e promove o desenvolvimento da capacidade de lidar com o sucesso e com o falhanço (Smith \& Smoll, 1996). Através do confronto com adversidades e exigências físicas e psicológicas, os jovens vão desenvolvendo atitudes importantes acerca da realização pessoal, da aceitação da autoridade e aprendem a ser persistentes face ao insucesso (Smith \& Smoll, 1990).

No entanto, a prática desportiva nem sempre é acompanhada de consequências positivas, estando, por vezes, relacionada com resultados indesejáveis (Holt \& Neely, 2011). Neste caso, a participação dos jovens em atividades desportivas pode tornar-se uma experiência stressante associada a emoções desagradáveis, sendo um dos exemplos mais evidentes a ansiedade competitiva que conduz ao sentimento de "medo de falhar", sendo responsável pela desvalorização e evitamento do desporto, por parte do atleta (Neil, Hanton, Mellalieu, \& Fletcher, 2011).

A razão pela qual a prática desportiva juvenil pode gerar estes diferentes resultados tem suscitado o interesse dos investigadores. A este nível, alguns autores chamam a atenção para o facto da simples participação dos jovens em contextos de formação desportiva não equivaler automaticamente a benefícios para $o$ desenvolvimento enquanto atletas e pessoas (Gomes, 2011; Petitpas, Cornelius, Van Raalte, \& Jones, 2005), sendo relevante estudar o modo como estes vivenciam os desafios e as exigências que lhes são colocadas pelo contexto desportivo. A título de exemplo, existem dados da investigação que demonstram que $\mathrm{o}$ desporto juvenil pode estar associado a consequências negativas para o bem-estar dos atletas, nomeadamente em termos da baixo autoestima, do desenvolvimento de comportamentos agressivos associados à baixa vontade de cooperar e competir com "fair-play" e da ansiedade excessiva relacionada com a elevada pressão por parte dos adultos (e.g., treinadores, pais, etc.) que pode levar a situações de stress competitivo e burnout (Côté \& Hay, 2002; Eklund \& Cresswell, 2007; Tremayne \& Tremayne, 2004).

Este estudo procura clarificar estes efeitos potenciais do desporto juvenil, centrando esta análise nos processos de avaliação cognitiva, nomeadamente no significado atribuído pelos jovens atletas à prática desportiva, analisando até que ponto esta é considerada como potencialmente geradora de ameaça ou de desafio para os atletas (Lazarus, 1991). Mais concretamente, este estudo procura, por um lado, verificar se diferentes padrões de avaliação cognitiva dos atletas (e.g., maior ou menor perceção de ameaça, maior ou menor perceção de desafio) correspondem a experiências distintas de ansiedade e de burnout (esgotamento) nos atletas e, por outro lado, procura verificar até que ponto a ansiedade sentida pelos atletas relativamente ao desporto e o modo como avaliam esta atividade podem explicar a sua experiência de burnout.

Tendo por base o primeiro objetivo, este estudo focou-se na análise dos processos de avaliação cognitiva, seguindo-se o Modelo Transacional Cognitivo, Motivacional e Relacional de Lazarus (1991; Lazarus \& Folkman, 1984). Segundo este modelo, o stress resulta de uma relação dinâmica, bidirecional e mutuamente recíproca entre o indivíduo (características pessoais) e o ambiente que o rodeia (características do contexto) (Lazarus, 1991; Lazarus \& Folkman, 1984). Assim, o stress não resulta apenas de fatores individuais ou de fatores ambientais, mas sim de uma transação entre ambos. Os estados psicológicos associados ao stress resultam de uma avaliação que o indivíduo faz acerca das competências que possui para lidar com as exigências da situação, sendo que, no caso dos estados 
psicológicos negativos (e.g., ansiedade), esta avaliação tem por base um desequilíbrio entre as exigências do ambiente e os recursos que a pessoa julga possuir para lidar com essas mesmas exigências (Lazarus \& Folkman, 1984).

Considerando o contexto desportivo, as reações de stress são desencadeadas quando o atleta perceciona a atividade desportiva como demasiado exigente, tendo em conta os recursos pessoais que possui para lidar com a situação. Este tipo de avaliação tem por base o conceito de "significado relacional" que diz respeito ao modo como o atleta encara a sua relação com o ambiente (Lazarus, 1991). Neste caso, as reações de stress dos atletas face às situações desportivas advêm, assim, dos processos de avaliação cognitiva que são os responsáveis pela interpretação das situações e pela maneira como estas vão ser geridas.

Segundo a perspetiva transacional de Lazarus (1991), a avaliação cognitiva divide-se em duas componentes: avaliação cognitiva primária e avaliação cognitiva secundária. A primária refere-se à interpretação da importância e do significado de determinado acontecimento para o bem-estar do indivíduo (Dias, Cruz, \& Fonseca, 2012). Quando um acontecimento é avaliado como stressante, podem ser feitas quatro tipos de avaliações: desafio, ameaça, benefício e perda ou dano (Dias, Cruz, \& Fonseca, 2012). A ameaça é a antecipação de consequências negativas, ou seja, é a avaliação da situação como perigosa para o bem-estar do indivíduo (potencial de perda), enquanto o desafio é a avaliação da situação como potencialmente benéfica para a pessoa (potencial de ganho), ainda que o objetivo final seja de difícil alcance (Lazarus, 2000). Dito de modo mais simples, a perceção de desafio ocorre quando o indivíduo se sente capaz de lidar com mestria face à situação stressante e a perceção de ameaça ocorre quando este antecipa danos iminentes para o seu bem-estar. Resumidamente, o desafio e a ameaça pressupõem acontecimentos futuros da vida do indivíduo, sendo que a perda e dano referem-se a acontecimentos psicológicos negativos que já pressupõem um determinado prejuízo para a pessoa, enquanto o benefício refere-se a acontecimentos psicológicos potencialmente positivos que também já implica um determinado ganho para a pessoa (Turner \& Jones, 2014). No entanto, é essencial realçar que este tipo de avaliações só acontecem caso o acontecimento em causa seja avaliado como importante ou pessoalmente significativo para o indivíduo (Gomes, 2014). Por sua vez, a avaliação cognitiva secundária diz respeito aos processos de coping disponíveis e utilizados pelo indivíduo para lidar com as situações percecionadas como ameaçadoras, prejudiciais ou desafiadoras (Turner \& Jones, 2014). Em suma, a avaliação cognitiva primária é responsável pela interpretação das respostas às situações, enquanto a avaliação cognitiva secundária determina as respostas que irão ocorrer face ao acontecimento stressante (Gomes, 2014).

A investigação atual tem-se centrado essencialmente no estudo da avaliação cognitiva secundária, ou seja, no confronto com situações de stress, nomeadamente, no tipo de estratégias de coping utilizadas em situações potencialmente geradoras de pressão para o indivíduo (Dias, Cruz, \& Fonseca, 2012; Folkman, Lazarus, Gruen, \& DeLongis, 1986; Jordet \& Elferink-Gemser, 2012). Em contrapartida, existem menos estudos acerca da importância dos processos de avaliação cognitiva primária no modo como os atletas avaliam o seu ambiente competitivo e, consequentemente, acerca dos efeitos da avaliação cognitiva primária nos estados psicológicos dos atletas. No entanto, os dados existentes têm vindo a reforçar a importância do modo como os atletas avaliam as situações exigentes em que se encontram. Por exemplo, Neil et al. (2011) num estudo com atletas adultos verificaram que perante as exigências do ambiente competitivo, os atletas tendiam a assumir padrões de avaliação cognitiva diferentes que originavam emoções distintas que eram modeladas pelo significado construído na relação entre o atleta e o seu ambiente. Mais concretamente, quando os atletas avaliavam as suas reações cognitivas e emocionais face aos stressores desportivos 
como negativas, então tendiam a ter comportamentos mais debilitadores do rendimento desportivo, essencialmente justificado por uma diminuição da sua perceção de controlo; pelo contrário, sempre que estas mesmas reações cognitivas e emocionais eram reinterpretadas como positivas, os atletas tendiam a ter comportamentos mais facilitadores do rendimento desportivo, essencialmente justificado por um aumento na concentração e no esforço perante a situação em causa.

Apesar destes resultados encorajadores, a análise da importância das diferentes cognições nos estados emocionais dos atletas é ainda pouco conhecida. Por conseguinte, este estudo pretende colmatar a escassa investigação na área do desporto, nomeadamente no desporto juvenil, focando-se essencialmente na importância dos processos de avaliação cognitiva primária nos estados psicológicos dos atletas, nomeadamente ao nível da ansiedade e do burnout.

A opção pela escolha da ansiedade e do burnout como variáveis a analisar em conjunto com os processos de avaliação cognitiva, devese à importância destas duas dimensões em contextos desportivos. Assim, relativamente à ansiedade, esta é entendida como uma emoção negativa desencadeada por um desequilíbrio entre as exigências do ambiente competitivo e os recursos que as pessoas julgam possuir para lidar com elas (Lazarus, 1991; Lazarus \& Folkman, 1984). Este desequilíbrio nasce de uma avaliação do ambiente competitivo como ameaçador ao bem-estar da pessoa. Simplificando, a ansiedade é uma resposta do indivíduo desencadeada por uma avaliação cognitiva de ameaça face a um determinado acontecimento, considerado relevante para a pessoa em causa. Dada a importância e prevalência da emoção de ansiedade no desporto, incluímos neste estudo uma medida de avaliação da ansiedade-traço (e.g., Escala de Ansiedade no Desporto-2 / Sport Anxiety Scale-2) baseada na Teoria Multidimensional da Ansiedade, que distingue três dimensões nesta emoção: (a) perturbação da concentração, que diz respeito a dificuldades em manter a concentração em aspetos relevantes das tarefas a realizar; (b) preocupação, relacionada com apreensão acerca de um possível mau rendimento e das respetivas consequências negativas que daí advenham; e (c) ansiedade somática, que se relaciona com os sintomas físicos experienciados pelos atletas, principalmente ao nível do estômago e dos músculos (Martens, Burton, Vealey, Bump, \& Smith, 1990; Smith, Smoll, Cumming, \& Grossbard, 2006). Apesar de inicialmente se considerar que os jovens atletas seriam incapazes de discriminar entre as três componentes da ansiedade (ansiedade somática, preocupação e perturbação da concentração), alguns estudos realizados no desporto juvenil demonstram o contrário, nomeadamente com a utilização da Sport Anxiety Scale-2 (Grossbard, Smith, Smoll, \& Cumming, 2009; Smith et al., 2006). O estudo de validação da SAS-2, realizado com atletas com idades compreendidas entre os 9 e os 14 anos, confirmou o modelo de três dimensões proposto, o que indica que os atletas conseguiram diferenciar a componente cognitiva da componente somática da ansiedade (Smith et al., 2006).

Uma das consequências negativas, e mais conhecidas, da exposição a elevados níveis de stress é a experiência de burnout, referindo-se à exaustão física e psicológica do indivíduo, que mantendo-se ao longo do tempo conduzem a um estado de esgotamento. Neste sentido, para este estudo foi selecionado o Questionário de Burnout para Atletas / Athlete Burnout Questionnaire (Raedeke \& Smith, 2001), como medida do nível de esgotamento. Neste instrumento, o fenómeno de burnout é entendido a partir da proposta de Maslach e Jackson (1984) que operacionalizaram o conceito em três dimensões: (a) exaustão emocional, descrita como cansaço extremo e sobrecarga emocional devidas a grandes exigências ocupacionais; (b) despersonalização, em que os indivíduos desenvolvem atitudes negativas e impessoais relativamente ao seu trabalho; e (c) baixa realização pessoal, caracterizada por sentimentos de desilusão e insatisfação dos trabalhadores face ao trabalho. 
Raedeke (1997) considerou necessária a alteração deste entendimento da experiência de burnout, ajustando-o ao contexto desportivo. Desta forma, a dimensão da exaustão emocional foi alargada e passou a incluir também a exaustão física; a dimensão da baixa realização pessoal foi formulada como realização pessoal reduzida, caracterizando-se por sentimentos de ineficácia e por uma tendência do atleta se autoavaliar negativamente relativamente ao seu rendimento e realizações pessoais; e por fim, a dimensão da despersonalização passou a designar-se por desvalorização da prática desportiva, caracterizando-se pelo desenvolvimento de atitudes negativas para com o desporto e pela perda de interesse por esta atividade (podendo ou não ocorrer o afastamento da prática desportiva) (Raedeke, Smith, Kenttä, Arce, \& Francisco, 2014).

A investigação tem sido vasta no estudo dos efeitos da ansiedade e do burnout no rendimento competitivo, porém poucos estudos têm sido efetuados no sentido de observar a relação entre a ansiedade e o burnout nos atletas (Cremades, Wated, \& Wiggins, 2011) e, ainda mais evidente, é escassa a investigação acerca do papel da avaliação cognitiva na determinação destas duas experiências psicológicas. Neste sentido, tornase importante compreender de que forma a ansiedade está relacionada com a experiência de burnout nos atletas, nomeadamente nos mais jovens (Cremades, Wated, \& Wiggins, 2011), sendo igualmente relevante estabelecer o papel dos processos de avaliação cognitiva na experiência de ansiedade e de burnout.

Como referido anteriormente, é pertinente desenvolver investigação acerca da influência dos processos de avaliação cognitiva primária no modo como os atletas se sentem no desporto, nomeadamente, no desporto juvenil. Mais concretamente, pouco se sabe acerca da influência da avaliação cognitiva na ansiedade e no burnout, ou seja, de que forma os processos de avaliação cognitiva primária estão relacionados com a ansiedade e com a experiência de burnout, por parte dos atletas. No sentido de colmatar a falta de estudos nesta área e sintetizando os objetivos já referidos, o presente estudo propõe-se a:

(a) Analisar a experiência de ansiedade, avaliação cognitiva e burnout em jovens atletas.

(b) Analisar as relações entre a ansiedade, a avaliação cognitiva e o burnout.

(c) Identificar diferenças nos níveis de ansiedade-traço e burnout em função dos processos de avaliação cognitiva primária.

(d) Observar as variáveis preditoras da experiência de burnout.

\section{MÉTODO}

Este estudo assumiu uma natureza transversal, sendo analisadas relações entre as variáveis em estudo, através da seleção de uma amostra de conveniência.

\section{Amostra}

Participaram neste estudo 711 atletas de formação desportiva (622 do sexo masculino, $87.5 \%$ e 89 do sexo feminino, $12.5 \%$ ), com idades compreendidas entre os 12 e os 19 anos $(M=14.77 ; \quad D P=1.86)$. Todos os participantes pertenciam a clubes desportivos do concelho de Guimarães, representando várias modalidades coletivas, nomeadamente futebol de $11(n=346,48.7 \%)$, voleibol ( $n=$ $89,12.5 \%)$, basquetebol $(n=77,10.8 \%)$, futebol de 7 ( $n=48,6.8 \%)$, râguebi ( $n=38$, $5.3 \%)$, futsal $(n=36,5.1 \%)$, andebol $(n=36$, $4.8 \%)$, pólo aquático $(n=28,3.9 \%)$ e hóquei em patins $(n=15,2.1 \%)$. Relativamente à divisão competitiva, 242 atletas (34.5\%) competiam nas divisões principais das respetivas modalidades, enquanto 459 atletas (65.5\%) competiam em divisões secundárias. Tendo em consideração o escalão competitivo, observou-se que 324 atletas (46.2\%) pertenciam ao escalão sub-14, 226 atletas (32.3\%) ao escalão sub-16, 146 atletas (20.9\%) ao escalão sub-18 e quatro atletas (0.6\%) pertenciam ao escalão sub-20. No que concerne aos resultados desportivos obtidos, 426 participantes $(63.8 \%)$ afirmaram não ter ganho nenhum título competitivo, enquanto 242 participantes $(36.2 \%)$ afirmaram ter ganho títulos nacionais e/ou distritais. Quanto aos 
anos de prática da modalidade, verificou-se uma variação entre um e 16 anos $(M=5.07$; $D P=3.16)$.

\section{Instrumentos}

Questionário Demográfico.

Este instrumento recolheu informações relativas ao sexo, idade, modalidade, divisão competitiva, escalão competitivo, títulos obtidos e anos de prática da modalidade.

Escala de Ansiedade no Desporto-2 (EAD-2, Smith, Smoll, Cumming, \& Grossbard, 2006; adaptação de Cruz \& Gomes, 2007).

Esta escala foi adaptada da Sport Anxiety Scale, $S A S-2$, representando um instrumento de avaliação multidimensional do traço de ansiedade competitiva. Este instrumento avalia as diferenças individuais no traço de ansiedade somática e em duas dimensões da ansiedade cognitiva (preocupação e perturbação da concentração), sendo composto por 15 itens distribuídos por três subescalas: ansiedade somática (cinco itens; $\alpha=0.85$ ), preocupação (cinco itens; $\alpha=0.86$ ) e perturbação da concentração (cinco itens; $\alpha=0.74$ ). Os participantes responderam aos itens segundo uma escala de "likert" de quatro pontos ( $1=$ nunca; 4 = quase sempre). A análise fatorial confirmatória demonstrou boas propriedades psicométricas do instrumento $\left(\chi^{2}(87\right.$ g.l. $)=$ 343.325, $p<0.001$; RMSEA $=0.064,90 \%$ C.I. [0.057; 0.072]; CFI $=0.939 ; \mathrm{NFI}=0.920$; $\mathrm{TLI}$ $=0.926$ ) (Bentler, 2007).

Escala de Avaliação Cognitiva (EAC, Gomes \& Teixeira, 2016).

A EAC tem por base o modelo transacional de Lazarus (1991; Lazarus \& Folkman, 1984) e avalia duas dimensões: a avaliação cognitiva primária e a avaliação cognitiva secundária. Para este estudo foi utilizada a dimensão da avaliação cognitiva primária, que inclui três subescalas: (a) perceção de importância atribuída pelo atleta à sua atividade desportiva (três itens; $\alpha=0.86$ ); (b) perceção de ameaça face à atividade desportiva (três itens; $\alpha=0.77$ ) e perceção de desafio face à atividade desportiva (três itens; $\alpha=0.72$ ). Os itens foram respondidos numa escala de "likert" de sete pontos (ex: 0 = nada importante; $3=$ mais ou menos; $6=$ muito importante). Pontuações mais elevadas nas subescalas indicam maior nível de importância da competição para o atleta, maior perceção de ameaça e maior perceção de desafio. A análise fatorial confirmatória demonstrou boas propriedades psicométricas do instrumento $\left(\chi^{2}(24\right.$ g.l. $)=$ 73.330, $p<0.001$; RMSEA $=0.054$, 90\% C.I. [0.040; 0.068]; CFI $=0.977$; NFI $=0.966$; TLI $=0.965)$ (Bentler, 2007).

Questionário de Burnout em Atletas (QBA, Raedeke \& Smith, 2001; adaptação de Gomes, 2013).

Esta escala foi adaptada do Athlete Burnout Questionnaire originalmente desenvolvido por Raedeke e Smith (2001), tendo por objetivo avaliar os níveis de burnout dos atletas no desporto. Este instrumento é composto por três dimensões: exaustão emocional/física ( $\alpha=$ $0.89)$, realização pessoal reduzida $(\alpha=0.66)$ e desvalorização da prática desportiva $(\alpha=0.85)$. $\mathrm{O}$ instrumento é composto por 15 itens distribuídos pelas três subescalas descritas (cada subescala é composta por cinco itens), tendo os itens sido respondidos numa escala de "likert" de cinco pontos $(1=$ Quase nunca a 5 $=$ Quase sempre). $\mathrm{A}$ análise fatorial confirmatória demonstrou boas propriedades psicométricas do instrumento $\left(\chi^{2}(85\right.$ g.l. $)=$ 410.282, $p<0.001$; RMSEA $=0.073,90 \%$ C.I. [0.066; 0.081]; CFI $=0.935$; NFI $=0.919$; TLI $=0.919$ ) (Bentler, 2007), embora recorrendo à necessidade de correlacionar os erros entre os itens 2 e 4 e os itens 1 e 14 (sendo o primeiro caso talvez explicado pelo facto de ambos os itens avaliarem sintomas de cansaço face ao desporto, enquanto no segundo caso poderá ser explicado pelo facto de ambos os itens avaliarem aspetos relacionados com o sucesso face ao desporto).

\section{Procedimentos}

Em primeiro lugar, estabeleceu-se uma parceria com "Guimarães: Cidade Europeia do Desporto 2013" que permitiu a realização dos contactos diretos com os clubes desportivos do concelho de Guimarães. Posteriormente, o 
estudo foi aprovado pela Comissão de Ética da Universidade a que pertencem os autores deste trabalho (ref. CEUM 030/2014). De seguida, procedeu-se ao contacto com os responsáveis dos clubes para verificação da disponibilidade de participação no estudo e respetiva marcação da data da recolha de dados.

A todos os participantes menores de idade foi entregue um consentimento informado para preenchimento por parte dos encarregados de educação, com informações acerca dos objetivos do estudo, do caráter voluntário de participação e garantindo a confidencialidade dos dados fornecidos pelo(a) atleta.

Antes de cada aplicação dos questionários, explicou-se sucintamente os objetivos do estudo aos atletas e deram-se as instruções necessárias ao preenchimento dos mesmos, salvaguardando $\mathrm{o}$ anonimato dos dados fornecidos. A taxa de retorno dos protocolos de avaliação foi de $77.2 \%$, tendo sido recebidos 711 protocolos dos 921 entregues.

\section{Análise Estatística}

Para efeitos de análise e tratamento estatístico dos dados foi utilizado o programa informático Statistical Package for Social Sciences (SPSS - Versão 22.0). Para analisar a experiência de ansiedade, avaliação cognitiva e burnout nos jovens atletas, procedeu-se à análise descritiva destas variáveis, observandose os valores médios e percentuais de cada dimensão, tendo por base os valores da escala de "likert". No caso da experiência de burnout, convém referir que os valores de corte usados neste estudo tiveram por base indicações sugeridas por alguns autores (Shirom, 1989; ver também Gomes, Montenegro, Peixoto, \& Peixoto, 2010) para o "Maslach Burnout Inventory", que é um dos instrumentos mais conhecidos para avaliar este fenómeno em diferentes classes profissionais (Maslach \& Jackson, 1984) e que esteve na base do desenvolvimento do QBA. Mais concretamente, neste estudo foram definidos os valores da escala "likert" inferiores ou iguais a dois ("raramente") para indicar baixos níveis de exaustão emocional/física, realização pessoal reduzida e desvalorização da prática desportiva e valores iguais ou superiores a quatro ("frequentemente") para indicar elevados níveis nestas mesmas dimensões.

De modo a analisar as associações entre as variáveis em estudo, recorreu-se ao cálculo dos coeficientes de correlação de Pearson. Para analisar diferenças nos níveis de ansiedadetraço e burnout em função dos processos de avaliação cognitiva, criaram-se os seguintes grupos de comparação: baixa importância $v s$ elevada importância; baixa ameaça $v s$ elevada ameaça e baixo desafio $v s$ elevado desafio (considerando a distribuição dos resultados dos atletas num percentil 20 e 80, ver Gomes \& Teixeira, 2013). Antes da realização destes testes, foram efetuadas análises exploratórias dos dados no sentido de testar os pressupostos da utilização de testes paramétricos, nomeadamente análises multivariadas de variância (MANOVA). Os testes paramétricos foram comparados com os testes não paramétricos correspondentes, observando-se o mesmo tipo de conclusões nos resultados obtidos. Neste sentido, optámos por apresentar os resultados obtidos com os testes paramétricos, visto serem mais robustos e permitirem usar análises multivariadas, reduzindo-se assim o número de testes a realizar e a probabilidade do erro Tipo 1 (FifeSchaw, 2006).

Por último, para analisar as variáveis pessoais e desportivas preditoras da experiência de burnout, recorremos às análises de regressão hierárquica (método "enter") constatando-se a ausência de problemas nos modelos testados.

O valor de significância adotado neste estudo foi de $95 \%(p<0.05)$.

\section{RESULTADOS}

\section{Estatísticas Descritivas das Variáveis em Estudo}

Começando pelos níveis de ansiedade experienciados pelos atletas, é de salientar que $61.1 \%(n=435)$ da amostra apresentou baixos níveis de perturbação da concentração, 4.3\% ( $n$ = 31) apresentou elevados níveis de perturbação da concentração, $45.9 \%(n=326)$ demonstrou um elevado nível de preocupação 
com a sua modalidade desportiva e $13.6 \%$ ( $n=$ 97) apresentou baixo nível de preocupação. Quanto à ansiedade somática, 70.2\% $(n=499)$ da amostra revelou baixos níveis de ansiedade somática e $5.8 \%(n=41)$ dos atletas manifestou elevados níveis nesta dimensão.

Relativamente à avaliação cognitiva, verificou-se que a esmagadora maioria dos atletas $(n=682)$ percecionou a atividade desportiva como muito importante (96.3\%), enquanto apenas $0.4 \%(n=3)$ manifestou baixos níveis de perceção de importância. $\mathrm{Na}$ dimensão perceção de ameaça, 84.8\% ( $n=$ 594) dos atletas considerou a sua modalidade desportiva pouco ameaçadora e $4.2 \%(n=29)$ considerou-a bastante ameaçadora. Quanto à perceção de desafio, 95.4\% $(n=671)$ dos atletas consideraram a sua modalidade altamente desafiadora, enquanto $1.5 \%(n=11)$ dos atletas consideraram-na muito pouco desafiadora.

No que se refere ao burnout, verificou-se que $54.4 \%(n=383)$ dos atletas manifestaram elevados níveis de realização pessoal, enquanto $0.7 \%(n=6)$ dos atletas demonstraram baixos níveis de realização pessoal. $\mathrm{Na}$ dimensão exaustão emocional/física, $61 \%(n=430)$ da amostra revelou baixos níveis nesta dimensão e $5.4 \%(n=39)$ manifestou níveis elevados nesta mesma dimensão. Quanto à desvalorização da prática desportiva, $75.5 \%(n=531)$ dos atletas apresentaram baixos níveis nesta dimensão, enquanto $4 \%(n=29)$ demonstraram níveis elevados nesta dimensão.

Os valores médios das dimensões avaliadas podem ser consultados na Tabela 1 .

Tabela 1

Médias, Desvios-Padrão e Correlações entre Ansiedade (EAD - 2), Avaliação Cognitiva (EAC) e Burnout (QBA)

\begin{tabular}{|c|c|c|c|c|c|c|c|c|c|}
\hline & M (DP) & 1 & 2 & 3 & 4 & 5 & 6 & 7 & 8 \\
\hline \multicolumn{10}{|l|}{ Ansiedade } \\
\hline $\begin{array}{l}\text { 1. EAD-2: Ansiedade cognitiva } \\
\text { perturbação da concentração }\end{array}$ & $1.78(0.58)$ & -- & & & & & & & \\
\hline $\begin{array}{l}\text { 2. EAD-2: Ansiedade cognitiva } \\
\text { preocupação }\end{array}$ & $2.79(0.79)$ & $0.40^{* * *}$ & -- & & & & & & \\
\hline $\begin{array}{l}\text { 3. EAD-2: Ansiedade somática } \\
\text { Avaliação cognitiva }\end{array}$ & $1.67(0.63)$ & $0.61^{* * *}$ & $0.41^{* * *}$ & -- & & & & & \\
\hline 4. EAC: Perceção de ameaça & $0.71(1.18)$ & $0.21^{* * *}$ & 0.04 & $0.17^{* * *}$ & -- & & & & \\
\hline $\begin{array}{l}\text { 5. EAC: Perceção de desafio } \\
\text { Burnout }\end{array}$ & $5.44(0.93)$ & -0.06 & $0.12^{* *}$ & 0.01 & $-0.14^{* * *}$ & -- & & & \\
\hline $\begin{array}{l}\text { 6. QBA: Realização pessoal } \\
\text { reduzida }\end{array}$ & $2.19(0.83)$ & $0.29^{* * *}$ & 0.01 & $0.18^{* * *}$ & $0.29^{* * *}$ & $-0.27^{* * *}$ & -- & & \\
\hline $\begin{array}{l}\text { 7. QBA: Exaustão } \\
\text { emocional/física }\end{array}$ & $2.10(0.71)$ & $0.33^{* * *}$ & $0.14^{* * *}$ & $0.37^{* * *}$ & $0.23^{* * *}$ & $-0.14^{* * *}$ & $0.47^{* * *}$ & -- & \\
\hline $\begin{array}{l}\text { 8. QBA: Desvalorização da prática } \\
\text { desportiva }\end{array}$ & $1.71(0.88)$ & $0.30^{* * *}$ & 0.02 & $0.27^{* * *}$ & $0.26^{* * *}$ & $-0.21^{* * *}$ & $0.65^{* * *}$ & $0.64^{* * *}$ & -- \\
\hline
\end{tabular}

\section{Correlações entre as Variáveis em Estudo}

Em relação às correlações entre a avaliação cognitiva, por um lado, e a ansiedade e o burnout, por outro lado, observou-se que a perceção de ameaça apresentou correlações positivas significativas com a perturbação da concentração, a ansiedade somática, a realização pessoal reduzida, a exaustão emocional/física e a desvalorização da prática desportiva (a magnitude destas correlações foram baixas a moderadas). Não se observou nenhuma correlação significativa entre a perceção de ameaça e a preocupação. Quanto à perceção de desafio, esta apresentou uma correlação negativa significativa com a realização pessoal reduzida, a exaustão emocional/física e a desvalorização da prática desportiva; além disso, a perceção de desafio apresentou uma correlação positiva significativa com a preocupação (a magnitude destas correlações foram baixas a moderadas). 
Quanto à relação entre a ansiedade e o burnout, verificaram-se correlações positivas significativas entre a perturbação da concentração e a realização pessoal reduzida, a exaustão emocional/física e a desvalorização da prática desportiva. De igual modo, observaramse correlações positivas significativas entre a preocupação e a exaustão emocional/física, o que indica que maior preocupação está associada a maior exaustão emocional/física. Finalmente verificou-se uma correlação positiva entre a ansiedade somática e as três dimensões do burnout, sugerindo que maiores índices de ansiedade somática estão associados a maior realização pessoal reduzida, exaustão emocional/física e maior desvalorização da prática desportiva (uma vez mais, a magnitude das correlações entre ansiedade e burnout foram baixas a moderadas). Os valores de correlação entre estas variáveis podem ser encontrados na Tabela 1.

\section{Diferenças na Ansiedade e no Burnout em Função da Avaliação Cognitiva}

Nesta parte do trabalho, procurámos verificar se existiriam diferenças na experiência de ansiedade e burnout (variáveis dependentes) em função dos processos de avaliação cognitiva primária (variável independente), definindo-se os grupos de baixa e elevada importância, baixa e elevada ameaça e baixo e elevado desafio.

No primeiro grupo de comparações, colocámos em análise a perceção de importância. Foram encontradas diferenças multivariadas na ansiedade, Wilks' $\lambda=0.98$, $F(3,603)=4.65, p=0.003, \eta^{2}=0.023$, tendo os testes univariados revelado diferenças significativas na dimensão preocupação da EAD-2. Desta forma, os atletas que apresentaram perceção de importância elevada demonstraram maior preocupação. Os resultados foram semelhantes, considerando o burnout, Wilks' $\lambda=0.87, F(3,596)=28.60, p$
$<0.001, \eta^{2}=0.13$. Neste caso, os testes univariados revelaram diferenças significativas em todas as dimensões do burnout, ou seja, atletas com perceção de importância baixa demonstraram menor realização pessoal, maior exaustão emocional/física e maior desvalorização da prática desportiva.

No segundo grupo de comparações, examinámos as diferenças em função da perceção de ameaça. Relativamente à ansiedade, foram encontradas diferenças multivariadas, Wilks' $\lambda=0.93, F(3,540)=$ 13.04, $p<0.001, \eta^{2}=0.068$, tendo os testes univariados demonstrado que os atletas com perceção de ameaça elevada evidenciaram maior perturbação da concentração e ansiedade somática. Observaram-se resultados semelhantes em relação ao burnout, Wilks' $\lambda=$ $0.89, F(3,536)=22.91, p<0.001, \eta^{2}=0.114$, sendo que atletas com perceção de ameaça elevada apresentaram menor realização pessoal, maior exaustão emocional/física e maior desvalorização da prática desportiva.

Por fim, no terceiro grupo de comparações analisámos a perceção de desafio. Foram encontradas diferenças significativas na ansiedade, Wilks' $\lambda=0.98, F(3,606)=5.27, p$ $=0.001, \eta^{2}=0.025$, e no burnout, Wilks' $\lambda=$ $0.91, F(3,601)=20.64, p<0.001, \eta^{2}=0.09$. $\mathrm{Na}$ ansiedade, os testes univariados demonstraram diferenças significativas na dimensão preocupação, ou seja, atletas com perceção de desafio elevada manifestaram maior preocupação. Em relação ao burnout, os testes univariados apresentaram diferenças significativas em todas as dimensões. Assim, atletas em que se observou menor perceção de desafio revelaram menor realização pessoal, maior exaustão emocional/física e maior desvalorização da prática desportiva. A Tabela 2 apresenta todos os resultados encontrados nestas análises. 
Tabela 2

Diferenças Significativas em função da Avaliação Cognitiva Primária (EAC)

\begin{tabular}{|c|c|c|c|c|c|c|c|c|}
\hline \multirow{2}{*}{ VARIÁVEL } & \multicolumn{2}{|c|}{ Baixa importância } & \multicolumn{2}{|c|}{ Elevada importância } & \multirow[b]{2}{*}{$g$} & \multirow[b]{2}{*}{$F$} & \multirow[b]{2}{*}{$p$} & \multirow[b]{2}{*}{$\eta 2$} \\
\hline & $M(D P)$ & $(n)$ & $M(D P)$ & $(n)$ & & & & \\
\hline EAD-2: Preocupação & $2.66(0.77)$ & (190) & $2.87(0.81)$ & $(417)$ & -2.63 & 9.36 & 0.002 & 0.015 \\
\hline ABQ: Real. pes. red. & $2.44(0.67)$ & (189) & $1.91(0.68)$ & $(411)$ & -1.83 & 77.96 & $<0.001$ & 0.115 \\
\hline ABQ: Exaustão & $2.17(0.91)$ & (189) & $1.97(0.91)$ & $(411)$ & -1.17 & 6.70 & 0.010 & 0.011 \\
\hline \multirow[t]{2}{*}{ ABQ: Desvalorização } & $2.05(0.92)$ & $(189)$ & $1.56(0.84)$ & $(411)$ & -0.74 & 41.97 & $<0.001$ & 0.066 \\
\hline & \multicolumn{2}{|c|}{ Baixa ameaça } & \multicolumn{2}{|c|}{ Elevada ameaça } & & & & \\
\hline EAD-2: Pert & $1.68(0.56)$ & $(388)$ & $2.00(0.59)$ & $(156)$ & -2.53 & 36.81 & $<0.001$ & 0.064 \\
\hline EAD-2: Ans. somáti. & $1.57(0.60)$ & $(388)$ & $1.84(0.66)$ & $(156)$ & -2.01 & 20.61 & $<0.001$ & 0.037 \\
\hline ABQ: Real. pes. red. & $1.90(0.63)$ & $(385)$ & $2.42(0.77)$ & $(155)$ & -2.66 & 62.05 & $<0.001$ & 0.103 \\
\hline ABQ: Exaustão & $1.85(0.87)$ & (385) & $2.34(0.94)$ & (155) & -1.65 & 33.11 & $<0.001$ & 0.058 \\
\hline \multirow[t]{2}{*}{ ABQ: Desvalorização } & $1.55(0.80)$ & $(385)$ & $2.06(1.02)$ & $(155)$ & -1.45 & 37.67 & $<0.001$ & 0.065 \\
\hline & \multicolumn{2}{|c|}{ Baixo desafio } & \multicolumn{2}{|c|}{ Elevado desafio } & & & & \\
\hline EAD-2: Preocupação & $2.66(0.77)$ & (208) & $2.85(0.81)$ & $(402)$ & -2.61 & 7.36 & 0.007 & 0.012 \\
\hline ABQ: Real. pes. red. & $2.40(0.70)$ & (207) & $1.94(0.67)$ & (398) & -1.82 & 61.11 & $<0.001$ & 0.092 \\
\hline ABQ: Exaustão & $2.21(0.85)$ & (207) & $1.93(0.91)$ & (398) & -1.21 & 13.13 & $<0.001$ & 0.021 \\
\hline ABQ: Desvalorização & $1.99(0.92)$ & (207) & $1.57(0.84)$ & (398) & -0.75 & 31.99 & $<0.001$ & 0.050 \\
\hline
\end{tabular}

\section{Variáveis Preditoras do Burnout}

Por último, procurámos compreender quais as variáveis pessoais e desportivas dos atletas e que dimensões da ansiedade e da avaliação cognitiva poderiam predizer os índices de burnout, nas suas três dimensões.

As variáveis preditoras entraram no modelo por ordem de importância na possível explicação da variável predita (burnout). Primeiro, foram introduzidas as variáveis pessoais e desportivas - sexo, divisão competitiva, escalão, títulos obtidos e anos de prática de competição (bloco 1); de seguida, foram introduzidas as dimensões da ansiedade - perturbação da concentração, preocupação e ansiedade somática (bloco 2); e, por último, foram introduzidas as dimensões da avaliação cognitiva - perceção de ameaça e perceção de desafio (bloco 3).

Em relação às dimensões da avaliação cognitiva, a perceção de importância serviu para selecionar para estas análises apenas os atletas que avaliaram a sua atividade desportiva como significativa, tendo-se estabelecido como "ponto de corte" o valor igual ou inferior a dois para retirar os participantes desta análise (ver Gomes \& Teixeira, 2013). Desta forma, foram eliminados sete casos da amostra. Os resultados do modelo de regressão podem ser consultados na Tabela 3.

Começando pela realização pessoal reduzida, no bloco 1 a variável escalão desportivo assumiu-se como preditora da experiência de burnout, explicando $3.7 \%$ da variância. Deste modo, a tendência para a realização pessoal reduzida foi predita pela pertença aos escalões desportivos sub-16, sub18 e sub- 20 . No bloco 2 , emergiu como preditor da realização pessoal reduzida a perturbação da concentração, explicando $12.1 \%$ da variância. Assim, a tendência para uma realização pessoal reduzida foi predita por maiores índices de perturbação da concentração. No último bloco, ambas as dimensões da avaliação cognitiva foram preditoras da realização pessoal reduzida, explicando o modelo $21.4 \%$ da variância. Neste caso, a tendência para a menor realização pessoal foi predita por maiores níveis de perceção de ameaça e menores níveis de perceção de desafio. De modo a controlar outliers, foi necessário retirar dois casos do modelo final obtido.

Quanto à exaustão emocional/física, no bloco 1 não foram encontradas variáveis preditoras desta dimensão do burnout. No segundo bloco, a exaustão foi predita por duas dimensões da ansiedade, explicando o modelo $19.1 \%$ da variância. Neste sentido, esta dimensão foi predita por maiores índices de perturbação da concentração e de ansiedade somática. No bloco 3, a exaustão emocional/física foi predita pelas duas dimensões da avaliação cognitiva do modelo, explicando $22.3 \%$ da variância. Neste caso, a 
tendência para a exaustão emocional/física foi predita por maiores níveis de perceção de ameaça e menores níveis de perceção de desafio. Também nesta situação foi necessário controlar outliers, retirando-se um total de seis casos do modelo final obtido.

No que se refere à predição da desvalorização da prática desportiva, no bloco 1 o escalão desportivo foi preditor desta dimensão do burnout, explicando o modelo $3.2 \%$ da variância. Deste modo, a tendência para a desvalorização da prática desportiva foi predita pela pertença aos escalões desportivos sub-16, sub-18 e sub-20. No segundo bloco, a desvalorização foi predita por todas as dimensões da ansiedade, explicando o modelo $17.5 \%$ da variância. Assim, a tendência para a desvalorização foi predita por maiores níveis de perturbação da concentração e de ansiedade somática e por menores níveis de preocupação. No último bloco, verificou-se que tanto a perceção de ameaça como a perceção de desafio foram variáveis preditoras da desvalorização, explicando $22.6 \%$ da variância. Desta forma, a tendência para a desvalorização da prática desportiva foi predita por maiores índices de perceção de ameaça e menores índices de perceção de desafio. Para controlo de outliers, retiraram-se 25 casos do modelo final.

Tabela 3

Modelos de Regressão para a Predição das Dimensões de Burnout

\begin{tabular}{|c|c|c|c|c|c|}
\hline Realização pessoal reduzida & $R^{2}\left(R^{2}\right.$ ajust. $)$ & $\beta$ & $\begin{array}{c}\beta \\
\text { IC } 95 \% \\
\end{array}$ & $t$ & $p$ \\
\hline Bloco 1: Variáveis pessoais e desportivas & $0.044(0.037) \quad(5,608) 5.66$ & & & & $<0.001$ \\
\hline Sexo ${ }^{\text {(a) }}$ & & -0.08 & {$[-0.33,0.01]$} & -1.83 & 0.068 \\
\hline Divisão competitiva ${ }^{(\mathrm{b})}$ & & -0.01 & {$[-0.13,0.11]$} & -0.15 & 0.883 \\
\hline Escalão $^{(c)}$ & & 0.18 & {$[0.14,0.36]$} & 4.40 & $<0.001$ \\
\hline Anos de prática de competição ${ }^{(\mathrm{d})}$ & & 0.03 & {$[-0.08,0.16]$} & 0.73 & 0.466 \\
\hline Títulos obtidos (e) & & -0.01 & {$[-0.15,0.11]$} & -0.32 & 0.753 \\
\hline Bloco 2: Dimensões da ansiedade & $0.129(0.117)(8,605) 11.18$ & & & & $<0.001$ \\
\hline EAD-2: Perturbação da concentração & & 0.25 & {$[0.20,0.43]$} & 5.24 & $<0.001$ \\
\hline EAD-2: Preocupação & & -0.03 & {$[-0.10,0.05]$} & -0.62 & 0.536 \\
\hline EAD-2: Ansiedade somática & & 0.07 & {$[-0.03,0.19]$} & 1.49 & 0.137 \\
\hline Bloco 3: Dimensões da avaliação cognitiva & $0.227(0.214)(10,603) 17.74$ & & & & $<0.001$ \\
\hline EAC: Perceção de ameaça & & 0.20 & {$[0.07,0.16]$} & 5.24 & $<0.001$ \\
\hline o de desafio & & -0.24 & {$[-0.24,0.13]$} & -6.45 & $<0.001$ \\
\hline Exaustão emocional/física & $R^{2}$ ( $R^{2}$ ajust. $)$ & $\beta$ & $\begin{array}{c}\beta \\
\text { IC } 95 \%\end{array}$ & $t$ & $p$ \\
\hline Bloco 1: Variáveis pessoais e desportivas & $0.013(0.004) \quad(5,604) 1.53$ & & & & 0.179 \\
\hline Sexo ${ }^{(a)}$ & & -0.08 & {$[-0.41,0.03]$} & -1.73 & 0.084 \\
\hline Divisão competitiva ${ }^{(b)}$ & & 0.02 & {$[-0.12,0.19]$} & 0.41 & 0.683 \\
\hline Escalão $^{(c)}$ & & 0.03 & {$[-0.08,0.20]$} & 0.82 & 0.411 \\
\hline Anos de pr & & 0.05 & {$[-0.06,0.24]$} & 1.21 & 0.227 \\
\hline Títulos obtidos (e) & & -0.05 & {$[-0.25,0.08]$} & -0.97 & 0.333 \\
\hline Bloco 2: Dimensões da ansied & $0.201(0.191)(8,601) 18.93$ & & & & $<0.001$ \\
\hline EAD-2: Perturbação da concentração & & 0.17 & {$[0.12,0.41]$} & 3.66 & $<0.001$ \\
\hline EAD-2: Preocupação & & -0.07 & {$[-0.16,0.02]$} & -1.61 & 0.109 \\
\hline EAD-2: Ansiedade somática & & 0.34 & {$[0.35,0.61]$} & 7.43 & $<0.001$ \\
\hline Bloco 3: Dimensões da avaliação cognitiva & $0.236(0.223)(10,599) 18.52$ & & & & $<0.001$ \\
\hline EAC: Perceção de ameaça & & 0.14 & {$[0.05,0.16]$} & 3.71 & $<0.001$ \\
\hline EAC: Perceção de desafio & & -0.12 & {$[-0.18,-0.05]$} & -3.28 & 0.001 \\
\hline
\end{tabular}

\section{DISCUSSÃO}

O principal objetivo deste estudo foi analisar o modo como os jovens atletas vivenciam a atividade desportiva. Esta análise foi efetuada atribuindo um papel primordial aos processos de avaliação cognitiva primária (que nos indicam o modo como os jovens atletas vivenciam a sua atividade desportiva), 
procurando-se observar as relações entre estes processos de avaliação e a experiência de ansiedade e de burnout. Mais concretamente pretendeu-se analisar os níveis médios das dimensões da ansiedade, avaliação cognitiva e burnout nos participantes, analisar as associações entre estas variáveis, identificar diferenças nos índices de ansiedade e burnout em função dos processos de avaliação cognitiva primária e, ainda, observar as variáveis preditoras da experiência de burnout.

Antes de iniciarmos as análises em estudo, observámos as estruturas fatoriais dos instrumentos utilizados. Os resultados obtidos refletiram as estruturas originais dos instrumentos e os valores do Alpha de Cronbach (fidelidade) assumiram níveis bastante aceitáveis, em praticamente todas as dimensões dos instrumentos, significando que estas medidas podem ser interessantes para avaliar o modo como os jovens experienciam a sua atividade desportiva.

Relativamente aos valores médios das variáveis, observou-se que os participantes apresentaram índices reduzidos nas dimensões de ansiedade (exceção para a dimensão preocupação com índices elevados para $45.9 \%$ da amostra) e nas dimensões de burnout. Estes resultados são congruentes com os estudos de Raedeke e Smith (2001) com nadadores adolescentes e jovens universitários, que apresentaram níveis baixos a moderados de burnout e ansiedade-traço. Nas dimensões da avaliação cognitiva primária, o principal dado a reter sugere que a generalidade dos atletas perceciona a sua atividade desportiva como importante, desafiadora e pouco ameaçadora, o que traduz a possibilidade do desporto juvenil poder contribuir para uma experiência positiva para o desenvolvimento destes jovens (Holt \& Neely, 2011).

No que concerne às relações entre as variáveis psicológicas, podemos afirmar que os resultados observados também vão de encontro a outros resultados obtidos na literatura. No que diz respeito às dimensões da escala de avaliação cognitiva primária salientam-se dois aspetos. Em primeiro lugar, verificaram-se associações positivas significativas entre a perceção de ameaça e as dimensões da escala de ansiedade (excetuando a dimensão "preocupação") e as dimensões da escala de burnout, o que significa que maior perceção de ameaça está relacionada com maior tendência para a experiência de ansiedade e de burnout. O padrão inverso foi verificado na associação negativa significativa entre a perceção de desafio e as dimensões de burnout, o que significa que avaliações de perceção de desafio estão associadas a consequências mais positivas do que negativas. Além destas associações verificou-se ainda uma associação positiva significativa entre a perceção de desafio e a preocupação, o que sugere que os atletas encararam a preocupação sentida face ao ambiente competitivo como desafiante e motivadora ao rendimento, ao invés de a interpretarem como algo negativo (ameaçador). Talvez esta explicação ajude a compreender os valores obtidos de preocupação dos atletas face ao desporto ( $45.9 \%$ com valores elevados). Seja como for, e de um modo geral, estes resultados refletiram as tendências esperadas face à perspetiva transacional de stress e das emoções (Lazarus, 1991; Lazarus \& Folkman, 1984). Quanto às dimensões do burnout, de salientar que as dimensões de realização pessoal reduzida, exaustão emocional/física e de desvalorização da prática desportiva apresentaram correlações positivas significativas com a ansiedade (excetuando a dimensão preocupação, no caso da realização pessoal reduzida e da desvalorização da prática desportiva). Estes resultados confirmam o princípio de que a experiência de burnout, traduzida pela maior exaustão emocional/física e desvalorização da prática desportiva, está associada a elevados níveis de ansiedade (Raedeke et al., 2014).

Relativamente à importância dos processos de avaliação cognitiva na experiência de ansiedade e burnout, os resultados confirmaram, uma vez mais, as premissas da perspetiva transacional de Lazarus e colaboradores (Lazarus, 1991; Lazarus \& Folkman, 1984). Ou seja, atletas com maior perceção de ameaça evidenciaram maior perturbação da concentração e ansiedade 
somática bem como maior burnout, enquanto atletas com maior perceção de desafio apresentaram maior preocupação (ao contrário do esperado) e menor burnout. Neste sentido, estes dados sugerem o potencial efeito dos processos de avaliação cognitiva nas respostas adaptativas ou não adaptativas dos atletas face a situações consideradas stressantes no desporto (Dias, 2005; Gomes, 2014; Lazarus, 1991, 2000; Lazarus \& Folkman, 1984; Martens et al., 1990).

As análises de regressão efetuadas também evidenciaram a importância das variáveis de ansiedade e de avaliação cognitiva primária na predição do burnout. No que se refere à exaustão emocional/física, esta foi predita pelos maiores níveis de níveis de perturbação da concentração e de perceção de ameaça e, inversamente, por menores níveis de perceção de desafio. Já a desvalorização da prática desportiva foi predita por maiores níveis de perturbação da concentração e de ansiedade somática, a que se juntou os maiores níveis de perceção de ameaça e, inversamente, menores níveis de perceção de desafio. A desvalorização da prática desportiva foi predita pelas três dimensões da ansiedade (maior perturbação da concentração e ansiedade somática e, contrariamente ao esperado, menores níveis de preocupação) e pelas duas dimensões da avaliação cognitiva (maior perceção de ameaça e menor perceção de desafio). De referir ainda que a pertença aos escalões desportivos de sub16 , sub-18 e sub-20 foi igualmente preditor da realização pessoal reduzida e da desvalorização da prática desportiva. Em síntese, tanto as dimensões da ansiedade (embora em termos diferentes em cada área do burnout) como da avaliação cognitiva primária, foram variáveis importantes para compreender a experiência negativa de burnout dos participantes neste estudo. Estes resultados corroboram a ideia de que a ansiedade representa uma variável explicativa do burnout e demonstram ainda que os processos de avaliação cognitiva ao nível primário desempenham um papel fulcral na predição deste fenómeno (Cremades et al., 2011; Raedeke \& Smith, 2001).
Em suma, os resultados desta investigação comprovam o papel central dos processos de avaliação cognitiva primária na maneira como os jovens atletas respondem às exigências da sua atividade desportiva e, por conseguinte, estão relacionados com a experiência de ansiedade e de burnout. A única exceção a este padrão de resultados prende-se com as relações estabelecidas entre a dimensão de ansiedade cognitiva da EAD-2, preocupação, cujos resultados foram imprevistos nas correlações entre as variáveis em estudo (e.g., maior perceção de desafio na escala de avaliação cognitiva correlacionou-se positivamente com a preocupação), nas análises multivariadas (e.g., atletas com maior perceção de desafio evidenciaram maior preocupação) e nas análises de regressão (e.g., a desvalorização da prática desportiva foi predita por menores níveis de preocupação). Estes dados são inesperados e difíceis de explicar face à literatura existente. A única explicação possível poderá ser enquadrada na formulação dos itens desta subescala da EAD-2 (e.g., "Eu preocupome com o facto de poder não jogar/competir ao meu melhor nível"), sendo de admitir que os atletas não tenham encarado estas afirmações como algo necessariamente negativo, mas antes como um sinal de motivação e valorização da prática desportiva (e.g., estar envolvido na modalidade poderá ter significado para os participantes deste estudo demonstrar vontade em evitar jogar ou competir a um nível inferior do pretendido). No entanto, trata-se apenas de uma hipótese, que carece de esclarecimento em futuras aplicações do instrumento junto de jovens atletas.

Independentemente deste padrão inesperado, e de um modo geral, os dados deste estudo apontam para a necessidade da investigação futura se centrar na compreensão dos processos cognitivos subjacentes à experiência de stress e burnout no desporto juvenil, procurando-se não apenas determinar as relações entre as variáveis mas também o modo como se influenciam mutuamente ao longo do tempo. Como os estudos sobre a influência da avaliação cognitiva nos estados emocionais de jovens atletas são relativamente 
escassos, sugere-se a continuação da investigação nesta área, tomando como ponto de referência os resultados obtidos neste trabalho e considerando, não só a avaliação cognitiva primária, mas também os recursos de coping que os atletas possuem para lidar com a situação (avaliação cognitiva secundária). Um maior fluxo de estudos nesta área permitirá, no futuro, o desenvolvimento de estratégias e programas de intervenção que tornem ainda mais positiva, a experiência psicológica dos jovens no desporto.

Apesar da relevância dos resultados obtidos, algumas limitações devem ser descritas neste estudo. A principal limitação prende-se com a escassez de estudos na área (como já foi referido anteriormente), pelo que não abundam dados na literatura que ajudem a compreender em maior detalhe os resultados encontrados, nomeadamente ao nível do desporto juvenil. Uma segunda limitação diz respeito à generalização da amostra. Apesar do número elevado de participantes, todos eles pertenciam à mesma zona geográfica existindo também uma maior participação de atletas do sexo masculino do que do sexo feminino. Por fim, este estudo seguiu uma metodologia transversal pelo que não podem ser feitas relações de causa-efeito entre as variáveis em estudo; além disso os valores das correlações entre as variáveis em estudo foram de uma magnitude baixa a moderada (Dancey \& Reidy, 2008) e os valores do tamanho do efeito nas análises multivariadas variaram entre moderados (em quase todos os casos) a baixos (em dois casos) (ver Ferguson, 2009).

Apesar destas limitações, este estudo contribui positivamente para o conhecimento do modo como os jovens atletas vivenciam a sua atividade desportiva, nomeadamente ao nível da ansiedade, da avaliação cognitiva e do burnout, realçando o papel fulcral dos processos de avaliação cognitiva no estudo dos estados psicológicos dos atletas.

\section{Agradecimentos:}

Nada a declarar.
Conflito de Interesses:

Nada a declarar.

\section{Financiamento:}

Este estudo foi apoiado pela "Guimarães-Cidade Europeia do Desporto 2013"

\section{REFERÊNCIAS}

Bentler, P. M. (2007). On tests and indices for evaluating structural models. Personality and Individual Differences, 42(5), 825-829. http://doi.org/10.1016/j.paid.2006.09.024

Côté, J., \& Hay, J. (2002). Children's involvement in sport: A developmental perspective. Em J. M. Silva \& D. E. Stevens (Eds.), Psychological Foundations of Sport (pp. 484-502). Boston: Allyn and Bacon.

Cremades, J. G., Wated, G., \& Wiggins, M. S. (2011). Multiplicative Measurements of a Trait Anxiety Scale as Predictors of Burnout. Measurement in Physical Education and Exercise Science, 15(3), 220-233. http://doi.org/10.1080/1091367X.2011.5943 56

Cruz, J. F., \& Gomes, A. R. (2007). Escala de Ansiedade no Desporto (EAD-2) - Versão para investigação. Universidade do Minho.

Dancey, C. P., \& Reidy, J. (2008). Estatística sem Matemática para Psicologia - 3.ed.: Usando SPSS para Windows. Porto Alegre: Artmed Editora.

Dias, C. (2005). Do stress e ansiedade às emoçôes no desporto: Da importância da sua compreensão à necessidade da sua gestão (Dissertação de doutoramento em Psicologia do Desporto). Instituto de Educação e Psicologia, Universidade do Minho, Braga.

Dias, C., Cruz, J. F., \& Fonseca, A. M. (2012). The relationship between multidimensional competitive anxiety, cognitive threat appraisal, and coping strategies: A multi-sport study. International Journal of Sport and Exercise Psychology, 10(1), 52-65. http://doi.org/10.1080/1612197X.2012.6451 31

Dias, C., Cruz, J. F., \& Fonseca, A. M. (2012). The relationship between multidimensional competitive anxiety, cognitive threat appraisal, and coping strategies: A multi-sport study. International Journal of Sport and Exercise Psychology, 10(1), 52-65. http://doi.org/10.1080/1612197X.2012.6451 31

Eklund, R. C., \& Cresswell, S. L. (2007). Athlete burnout. Em G. Tenenbaum \& R. C. Eklund (Eds.), Handbook of Sport Psychology (pp. 621-641). Hoboken, New Jersey: John Wiley \& Sons.

Ferguson, C. J. (2009). An effect size primer: A guide for clinicians and researchers. Professional 
Psychology: Research and Practice, 40(5), 532538. http://doi.org/10.1037/a0015808

Fife-Schaw, C. (2006). Levels of measurement. Em G. M. Breakwell, S. Hammond, C. Fife-Schaw, \& J. A. Smith (Eds.), Research Methods in Psychology (3rd ed., pp. 50-63). London: SAGE.

Folkman, S., Lazarus, R. S., Gruen, R. J., \& DeLongis, A. (1986a). Appraisal, coping, health status, and psychological symptoms. Journal of Personality and Social Psychology, 50(3), 571-579.

Folkman, S., Lazarus, R. S., Gruen, R. J., \& DeLongis, a. (1986b). Appraisal, coping, health status, and psychological symptoms. Journal of personality and social psychology, 50(3), 571-9.

Gomes, A. R. (2011). A iniciação e formação desportiva e o desenvolvimento psicológico de crianças e jovens. Em A. A. Machado \& A. R. Gomes (Eds.), Psicologia do esporte: Da escola à competição (pp. 19-48). Várzea paulista: Editora Fontoura.

Gomes, A. R. (2013). Questionário de Burnout em Atletas (QBA) (Relatório técnico não publicado). Braga: Escola de Psicologia, Universidade do Minho.

Gomes, A. R. (2014). Positive human functioning in stress situations: An interactive proposal. Em A. R. Gomes, R. Resende, \& A. Albuquerque (Eds.), Positive human functioning from a multidimensional perspective: Promoting stress adaptation (Vol. 1, pp. 165-194). New York: Nova Science Publishers, Incorporated.

Gomes, A. R., Montenegro, N., Peixoto, A. M. B. da C., \& Peixoto, A. R. B. da C. (2010). Stress ocupacional no ensino: um estudo com professores dos $3^{\circ}$ ciclo e ensino secundário. Psicologia \& Sociedade, 22(3), 587-597.

Gomes, A. R., \& Teixeira, F. (2013). Influência dos processos de avaliação cognitiva na atividade laboral de bombeiros portugueses. Psico-USF, 18(2), 309-320.

Gomes, A. R., \& Teixeira, P. M. (2016). Stress, Cognitive Appraisal and Psychological Health: Testing Instruments for Health Professionals. Stress and Health: Journal of the International Society for the Investigation of Stress, 32(2), 167-172. http://doi.org/10.1002/smi.2583

Grossbard, J. R., Smith, R. E., Smoll, F. L., \& Cumming, S. P. (2009a). Competitive anxiety in young athletes: differentiating somatic anxiety, worry, and concentration disruption. Anxiety, Stress, and Coping, 22(2), 153-166. http://doi.org/10.1080/10615800802020643

Grossbard, J. R., Smith, R. E., Smoll, F. L., \& Cumming, S. P. (2009b). Competitive anxiety in young athletes: differentiating somatic anxiety, worry, and concentration disruption. Anxiety, stress, and coping, 22(2), 153-66. http://doi.org/10.1080/10615800802020643
Holt, N. L., \& Neely, K. C. (2011). Positive youth development trough sport: A review. Revista de Iberoamericana de Psicología Del Ejercicio Y El Deporte, 6(2), 299-316.

Jordet, G., \& Elferink-Gemser, M. T. (2012a). Stress, Coping, and Emotions on the World Stage: The Experience of Participating in a Major Soccer Tournament Penalty Shootout. Journal of Applied Sport Psychology, 24(1), 73-91. http://doi.org/10.1080/10413200.2011.61900 0

Jordet, G., \& Elferink-Gemser, M. T. (2012b). Stress, Coping, and Emotions on the World Stage: The Experience of Participating in a Major Soccer Tournament Penalty Shootout. Journal of Applied Sport Psychology, 24(1), 73-91. http://doi.org/10.1080/10413200.2011.61900 0

Lazarus, R. S. (1991). Emotion and Adaptation. New York: Oxford University Press.

Lazarus, R. S. (2000). How emotions influence performance in competitive sports. The Sport Psychologist, 14(3), 229-252.

Lazarus, R. S., \& Folkman, S. (1984). Stress, Appraisal, and Coping. New York: Springer Publishing Company.

Martens, R., Burton, D., Vealey, R. S., Bump, L. A., \& Smith, D. E. (1990). Development and validation of the competitive state anxiety inventory - 2 (CSAI-2). Em R. Martens, R. S. Vealey, \& D. Burton (Eds.), Competitive Anxiety in Sport (pp. 117-213). Champaign Ilinois: Human Kinetics.

Maslach, C., \& Jackson, S. E. (1984). Burnout in organizational settings. Em S. Oskamp (Ed.), Applications in organizational settings (Vol. 5, pp. 133-153). Beverly Hills: Sage Publications.

Neil, R., Hanton, S., Mellalieu, S. D., \& Fletcher, D. (2011). Competition stress and emotions in sport performers: The role of further appraisals. Psychology of Sport and Exercise, 12(4), 460470.

http://doi.org/10.1016/j.psychsport.2011.02.0 01

Petitpas, A. J., Cornelius, A. E., Van Raalte, J. L., \& Jones, T. (2005). A framework for planning youth sport programs that foster psychosocial development. The Sport Psychologist, 19(3), 63-80.

Raedeke, T. D. (1997). Is athlete burnout more than just stress? A sport commitment perspective. Journal of Sport \& Exercise Psychology, 10(4), 396-417.

Raedeke, T. D., \& Smith, A. L. (2001). Development and preliminary validation of an athlete burnout measure. Journal of Sport \& Exercise Psychology, 23(4), 281-306.

Raedeke, T. D., Smith, A. L., Kenttä, G., Arce, C., \& Francisco, C. (2014). Burnout in sport: From theory to intervention. Em A. R. Gomes, R. Resende, \& A. Albuquerque (Eds.), Positive human functioning from a multidimensional 
perspective: Promoting stress adaptation (Vol. 1, pp. 113-141). New York: Nova Science Publishers, Incorporated.

Shirom, A. (1989). Burnout in work organizations. Em C. L. Cooper \& I. Robertson (Eds.), International Review of Industrial and Organizational Psychology (pp. 25-48). New York: John Wiley \& Sons.

Smith, R. E., \& Smoll, F. L. (1990). Sport performance anxiety. Em H. Leitenberg (Ed.), Handbook of Social and Evaluation Anxiety (pp. 417-454). New York: Plenum Press.

Smith, R. E., \& Smoll, F. L. (1996). Psychosocial interventions in youth sport. Em J. L. V. Raalte \& B. W. Brewer (Eds.), Exploring Sport and Exercise Psychology (pp. 287-315). Washington DC: American Psychological Association.

Smith, R. E., Smoll, F. L., Cumming, S. P., \& Grossbard, J. R. (2006). Measurement of multidimensional sport performance anxiety in children and adults: The sport anxiety scale- 2 .
Journal of Sport \& Exercise Psychology, 28(4), 479-501.

Smith, R. E., Smoll, F. L., Cumming, S. P., \& Grossbard, J. R. (2006). Measurement of Multidimensional Sport Performance Anxiety in Children and Adults: The Sport Anxiety Scale-2. Journal of Sport \& Exercice Psychology, 28, 479-501.

Tremayne, P., \& Tremayne, B. (2004). Children and sport psychology. Em T. Morris \& J. Summers (Eds.), Sport Psychology: Theories, Applications and Issues (2nd ed.). Milton: Wiley.

Turner, M. J., \& Jones, M. (2014). Stress, emotions and athletes' positive adapation to sport: Contributions from a transactional perspective. Em A. R. Gomes, R. Resende, \& A. Albuquerque (Eds.), Positive human functioning from a multidimensional perspective: Promoting stress adaptation (Vol. 1, pp. 85-111). New York: Nova Science Publishers, Incorporated. 